\title{
The existence and nonexistence of
} positive solutions for fractional differential equations with nonhomogeneous boundary conditions

\author{
Xiaofeng Su, Mei Jia* and Mengmeng Li
}

${ }^{*}$ Correspondence:

jiamei-usst@163.com

College of Science, University of

Shanghai for Science and

Technology, Shanghai, 200093,

China

\begin{abstract}
In this paper, we study the existence and nonexistence of the positive solutions for a class of fractional differential equations with nonhomogeneous boundary conditions and the impact of the disturbance parameters $a, b$ on the existence of positive solutions. By using the upper and lower solutions method and the Schauder fixed point theorem, we obtain the sufficient conditions for the boundary value problem to have at least one positive solution, two positive solutions, and no positive solution, respectively. Moreover, under certain conditions, we prove that there exists a bounded and continuous curve $L$ dividing $[0,+\infty) \times[0,+\infty)$ into two separate subsets $\Lambda^{E}$ and $\Lambda^{N}$ with $L \subseteq \Lambda^{E}$ such that the boundary value problem has at least two positive solutions for each $(a, b) \in \Lambda^{E} \backslash L$, one positive solution for each $(a, b) \in L$, and no positive solution for any $(a, b) \in \Lambda^{N}$. Finally, we give some examples to illustrate our main results.
\end{abstract}

MSC: $34 \mathrm{~B} 15 ; 26 \mathrm{~A} 33$

Keywords: fractional differential equations; existence and nonexistence; positive solutions; upper and lower solution method; fixed point theorem

\section{Introduction}

In recent years, since the fractional differential equation has been widely applied in various areas such as mathematics, physics, chemistry, biology, and so forth, lots of books on fractional calculus have appeared, see [1-4] and the references therein. The boundary value problems are a very important part of fractional differential equations theory, and more and more researchers' attention has been attracted and plenty of meaningful results have been obtained; see [5-23]. For example, in [21], the authors studied the existence of multiple positive solutions of systems of the boundary value problems of the Caputo fractional differential equations

$$
\begin{cases}-{ }^{C} D^{q} z_{i}(t)=f_{i}(t, z(t)), & \text { for a.e. } t \in[0,1] \text { and } i \in I_{n}, \\ \alpha z_{i}(0)-\beta z_{i}^{\prime}(0)=0, & \gamma z_{i}(1)+\delta z_{i}^{\prime}(1)=0,\end{cases}
$$

(c) 2016 Su et al. This article is distributed under the terms of the Creative Commons Attribution 4.0 International License (http://creativecommons.org/licenses/by/4.0/), which permits unrestricted use, distribution, and reproduction in any medium, provided you give appropriate credit to the original author(s) and the source, provide a link to the Creative Commons license, and indicate if changes were made. 
where $\mathbf{z}(t)=\left(z_{1}(t), \ldots, z_{n}(t)\right), q \in(1,2)$, the parameters $\alpha, \beta, \gamma, \delta$ are positive real numbers, and obtained some interesting conclusions. Reference [22] studied the existence of at least one positive solution for the following problem:

$$
\left\{\begin{array}{l}
D_{0^{+}}^{\alpha} u(t)=f(t, u(t)), \quad t \in(0, \infty), \\
\lim _{t \rightarrow 0^{+}} t^{2-\alpha} u(t)=a, \quad \lim _{t \rightarrow 0^{+}} D_{0^{+}}^{\alpha-1} u(t)=b
\end{array}\right.
$$

where $1<\alpha \leq 2, a, b \in \mathbb{R}, f:(0, \infty) \times \mathbb{R} \rightarrow \mathbb{R}$ is a continuous function, and $f$ may be singular at $t=0$, i.e., $\lim _{t \rightarrow 0^{+}} f(t, \cdot)=+\infty$. And the paper also considered an infinite fractional boundary value problem for singular integro-differential equation of mixed type on the half line

$$
\left\{\begin{array}{l}
D_{0^{+}}^{\alpha} u(t)=f(t, u(t),(S u)(t),(H u)(t)), \quad t \in(0, \infty), \\
\lim _{t \rightarrow 0^{+}} t^{2-\alpha} u(t)=a, \quad \lim _{t \rightarrow 0^{+}} D_{0^{+}}^{\alpha-1} u(t)=b,
\end{array}\right.
$$

where $S, H:(0, \infty) \times(0, \infty) \rightarrow[0, \infty),(S u)(t)=\int_{0}^{\infty} K(t, s) u(s) \mathrm{d} s,(H u)(t)=\int_{0}^{t} H(t, s) u(s) \mathrm{d} s$. The authors proved the existence of at least one positive solution for two problems of the boundary value problem for the fractional differential equation by using a fixed point theorem in partially ordered sets and the contraction mapping principle.

The disturbance parameter in boundary conditions is a very important factor while solving the actual problems with the boundary value problems of differential equations and also it is inevitable that the impact of such a disturbance parameter to the existence of solutions always exists. However, as far as we know, there are only several papers studying this kind of impact, see [23-28]. In [24], the authors studied the second-order differential equation boundary value problem with one-parameter nonhomogeneous boundary conditions

$$
\left\{\begin{array}{l}
u^{\prime \prime}+p(t) u^{\prime}+g(t)=0, \quad t \in(0,1) \\
u(0)=0, \quad u(1)=\lambda
\end{array}\right.
$$

And the multi-point boundary value problem

$$
\left\{\begin{array}{l}
u^{\prime \prime}(t)+a(t) f(u)=0, \quad t \in(0,1), \\
u^{\prime}(0)=0, \quad u(1)-\sum_{i=1}^{n-2} k_{i} u\left(\xi_{i}\right)=b
\end{array}\right.
$$

was considered in [25]. References [26, 27] studied the two-parameter nonhomogeneous boundary value problem of the second-order differential equation

$$
\left\{\begin{array}{l}
u^{\prime \prime}(t)=f\left(t, u, u^{\prime}\right), \quad t \in(0,1), \\
u(0)-\sum_{i=1}^{m} a_{i} u\left(t_{i}\right)=\lambda_{1}, \quad u(1)-\sum_{i=1}^{m} b_{i} u\left(t_{i}\right)=\lambda_{2} .
\end{array}\right.
$$

It was shown that under some assumptions there exists a continuous curve $\Gamma$ separating the plane $\mathbb{R}^{2}$ into disjoint regions $\Lambda^{E}$ and $\Lambda^{N}$ with $\Gamma \subseteq \Lambda^{E}$ such that this boundary value 
problem has at least two solutions on $\Lambda^{E} \backslash \Gamma$, one solution on $\Gamma$, and no solution on $\Lambda^{N}$. The authors also studied the higher order boundary value problems with nonhomogeneous boundary conditions in [28] and get the some conclusion. Reference [23] studied the existence and nonexistence of positive solutions for the boundary value problems of the fractional differential equations

$$
\left\{\begin{array}{l}
-{ }^{C} D^{\delta} u(t)=f(t, u(t)), \quad t \in(0,1), \\
m_{1} u(0)-n_{1} u^{\prime}(0)=0, \\
m_{2} u(1)+n_{2} u^{\prime}(1)=\int_{0}^{1} g(s) u(s) \mathrm{d} s+a,
\end{array}\right.
$$

where ${ }^{C} D^{\delta}$ is the Caputo fractional derivative of order $\delta$. And one studied the impact of the disturbance parameter $a$ on the existence of positive solutions. Under certain conditions, the authors also obtained the demarcation point which divides the disturbance parameters into two subintervals such that the boundary value problem has positive solutions for the disturbance parameter in one subinterval while there are no positive solutions in the other.

In this paper, we study the existence and nonexistence of the positive solutions of the fractional order the boundary value problem which is composed of the equation

$$
D_{0^{+}}^{\delta} u(t)+f(t, u(t))=0, \quad \text { for a.e. } t \in(0,1)
$$

and nonhomogeneous boundary conditions with the disturbance parameters $a, b$

$$
\lim _{t \rightarrow 0^{+}} t^{2-\delta} u(t)=a, \quad u(1)=b,
$$

where $D_{0^{+}}^{\delta}$ is the standard Riemann-Liouville fractional derivative of order $\delta, 1<\delta<2, J=$ $(0,1]$, disturbance parameters $a \geq 0, b \geq 0, f:[0,1] \times[0,+\infty) \rightarrow[0,+\infty)$, and $f$ satisfies the $L_{1}$-Caratheódory conditions.

We say that $f$ satisfies the $L_{1}$-Caratheódory conditions on $[0,1] \times[0,+\infty)$, if

(i) $f(\cdot, u)$ is measurable for all $u \in[0,+\infty)$;

(ii) $f(t, \cdot)$ is continuous for a.e. $t \in[0,1]$;

(iii) for every $\gamma>0$, there exists $\varphi_{\gamma} \in L_{1}[0,1]$ such that $\left|f\left(t, t^{\delta-2} u\right)\right| \leq \varphi_{\gamma}(t)$ for all $u \in[0, \gamma]$ and a.e. $t \in[0,1]$.

In the paper, we have the following.

(H) For any $t \in J, f(t, u)$ is monotone increasing with respect to $u$.

We focus on studying the impact of the disturbance parameters $a, b$ on the existence of positive solutions. We use the upper and lower solutions method and the Schauder fixed point theorem, and we obtain sufficient conditions so that the problem has at least one positive solution, two positive solutions, and no positive solution, respectively. In addition, we also prove that there exists a bounded and continuous curve $L$ dividing $[0,+\infty) \times[0,+\infty)$ into two separate subsets $\Lambda^{E}$ and $\Lambda^{N}$ with $L \subseteq \Lambda^{E}$ such that the boundary value problem has at least two positive solutions for each $(a, b) \in \Lambda^{E} \backslash L$, one positive solution for each $(a, b) \in L$, and no positive solution for any $(a, b) \in \Lambda^{N}$ under certain conditions. 


\section{Preliminaries}

In this section, we present some useful definitions and the related theorems.

Definition 2.1 (See [4]) Let $u \in L_{1}[0,1]$. The integrals

$$
I_{0^{+}}^{p} u(t)=\frac{1}{\Gamma(p)} \int_{0}^{t} \frac{u(s)}{(t-s)^{1-p}} \mathrm{~d} s, \quad t>0,
$$

where $p>0$, is called the Riemann-Liouville fractional integral of order $p$.

Definition 2.2 (See [4]) The Riemann-Liouville derivative of order $p$ for a continuous function $u:(0,+\infty) \rightarrow \mathbb{R}$ is given by

$$
D_{0^{+}}^{p} u(t)=\frac{1}{\Gamma(n-p)}\left(\frac{d}{d t}\right)^{n} \int_{0}^{t} \frac{u(s)}{(t-s)^{p+1-n}} \mathrm{~d} s=\left(\frac{d}{d t}\right)^{n} I_{0^{+}}^{n-p} u(t),
$$

provided the right side is pointwise defined on $(0,+\infty)$, where $n=[p]+1$ and $n-1<p<n$.

Lemma 2.1 (See [3], Lemma 1.1) The space $A C^{n}[0,1]\left(n \in \mathbb{N}_{+}\right)$consists of those and only those functions $u(t)$ which can be represented in the form

$$
u(t)=\left(I_{0^{+}}^{n} y\right)(t)+\sum_{k=0}^{n-1} c_{k} t^{k},
$$

where $y(t) \in L_{1}[0,1], c_{k}(k=0,1, \ldots, n-1)$ are arbitrary constants, and

$$
\left(I_{0^{+}}^{n} y\right)(t)=\frac{1}{(n-1) !} \int_{0}^{t}(t-s)^{n-1} y(s) \mathrm{d} s .
$$

Lemma 2.2 (See [3], Lemma 2.4) Let $p>0$. If $u \in L_{1}[0,1]$, then the equality

$$
\left(D_{0^{+}}^{p} I_{0^{+}}^{p} u\right)(t)=u(t)
$$

holds a.e. on $[0,1]$.

Lemma 2.3 (See [3], Corollary 2.1) Let $p>0$ and $n=[p]+1$, the equality $\left(D_{0^{+}}^{p} u\right)(t)=0$ is valid if, and only if,

$$
u(t)=\sum_{k=1}^{n} c_{k} t^{p-k}
$$

where $c_{k} \in \mathbb{R}(k=1,2, \ldots)$ are arbitrary constants.

Lemma 2.4 (See [3], Lemma 2.5) Let $p>0$ and $n=[p]+1$. If $u \in L_{1}[0,1]$ and $I_{0^{+}}^{n-p} \in$ $A C^{n}[0,1]$, then the equality

$$
I_{0^{+}}^{p}\left(D_{0^{+}}^{p} u\right)(t)=u(t)-\sum_{k=1}^{n} c_{k} t^{p-k}
$$

holds a.e. on $[0,1]$. 
Let

$$
E:=C_{2-\delta}^{0}[0,1]=\left\{u \in C(0,1]: \lim _{t \rightarrow 0^{+}} t^{2-\delta} u(t)<+\infty\right\}
$$

then $E$ is a Banach space with the norm $\|u\|=\sup _{t \in[0,1]} t^{2-\delta}|u(t)|$.

Lemma 2.5 If $D_{0^{+}}^{\delta} u \in L_{1}[0,1]$, then $I_{0^{+}}^{2-\delta} u \in A C^{2}[0,1]$.

Proof Since $D_{0^{+}}^{\delta} u \in L_{1}[0,1]$ and $\left(D_{0^{+}}^{\delta} u\right)(t)=\left(\frac{d}{d t}\right)^{2} I_{0^{+}}^{2-\delta} u(t)$, it follows that $I_{0^{+}}^{2-\delta} u \in A C^{2}[0,1]$ from Lemma 2.1.

Definition 2.3 If $u \in E, D_{0^{+}}^{\delta} u \in L_{1}[0,1]$ and satisfies (1.1) and (1.2), $u$ is called a solution of the fractional boundary value problem (1.1) and (1.2). Furthermore, if $u(t)>0, t \in(0,1)$, $u$ is called a positive solution of the fractional boundary value problem (1.1) and (1.2).

Lemma 2.6 For any $y \in L_{1}[0,1]$, the boundary value problem

$$
\begin{aligned}
& D_{0^{+}}^{\delta} u(t)+y(t)=0, \quad \text { for a.e. } t \in(0,1), \\
& \lim _{t \rightarrow 0^{+}} t^{2-\delta} u(t)=a, \quad u(1)=b,
\end{aligned}
$$

has a unique solution

$$
u(t)=\int_{0}^{t} G(t, s) y(s) \mathrm{d} s+(b-a) t^{\delta-1}+a t^{\delta-2}
$$

where

$$
G(t, s)=\frac{1}{\Gamma(\delta)} \begin{cases}(t(1-s))^{\delta-1}-(t-s)^{\delta-1}, & 0 \leq s \leq t \leq 1 \\ (t(1-s))^{\delta-1}, & 0 \leq t \leq s \leq 1\end{cases}
$$

Proof Suppose $u$ is a solution of the boundary value problem (2.1) and (2.2). Since $y \in$ $L_{1}[0,1],(2.1)$ means that there exists a.e. on $[0,1]$ the fractional derivative $D_{0^{+}}^{\delta} u \in L_{1}[0,1]$. By Lemma $2.5, I_{0^{+}}^{2-\delta} u(t) \in A C^{2}[0,1]$. Thus we can apply Lemma 2.4 , we have

$$
\left(I_{0^{+}}^{\delta} D_{0^{+}}^{\delta} u\right)(t)=u(t)-\sum_{j=1}^{2} c_{j} t^{\delta-j}
$$

that is,

$$
u(t)=-I^{\delta} y(t)+c_{0} t^{\delta-1}+c_{1} t^{\delta-2}
$$

By the boundary conditions $\lim _{t \rightarrow 0^{+}} t^{2-\delta} u(t)=a$ and $u(1)=b$, we can show

$$
c_{0}=\frac{1}{\Gamma(\delta)} \int_{0}^{1}(1-s)^{\delta-1} y(s) \mathrm{d} s+(b-a), \quad c_{1}=a .
$$


Then

$$
\begin{aligned}
u(t)= & -\frac{1}{\Gamma(\delta)} \int_{0}^{t}(t-s)^{\delta-1} y(s) \mathrm{d} s+\frac{1}{\Gamma(\delta)} \int_{0}^{1}(t(1-s))^{\delta-1} y(s) \mathrm{d} s+(b-a) t^{\delta-1}+a t^{\delta-2} \\
= & \frac{1}{\Gamma(\delta)} \int_{0}^{t}\left((t(1-s))^{\delta-1}-(t-s)^{\delta-1}\right) y(s) \mathrm{d} s+\frac{1}{\Gamma(\delta)} \int_{t}^{1}(t(1-s))^{\delta-1} y(s) \mathrm{d} s \\
& +a t^{\delta-2}-a t^{\delta-1}+b t^{\delta-1} \\
= & \int_{0}^{1} G(t, s) y(s) \mathrm{d} s+(b-a) t^{\delta-1}+a t^{\delta-2} .
\end{aligned}
$$

On the other hand, if $u$ satisfies the (2.3), then $u$ satisfies the (2.5), too. Applying the operator $D_{0^{+}}^{\delta}$ to both sides of (2.5), we have

$$
\left(D_{0^{+}}^{\delta} u\right)(t)=\left(\frac{d}{d t}\right)^{2} I_{0^{+}}^{2-\delta} u(t)=-\left(\frac{d}{d t}\right)^{2}\left(I_{0^{+}}^{2-\delta} I_{0^{+}}^{\delta} y\right)(t)=-\left(\frac{d}{d t}\right)^{2} I_{0^{+}}^{2} y(t)=-y(t),
$$

which implies (2.1) is satisfied.

We can easily show that $u$ satisfies (2.2).

Lemma 2.7 Let $G$ be defined by (2.4). Then,

(1) for any $t, s \in(0,1), G(t, s)>0$;

(2) for any $t, s \in[0,1], G(t, s)$ is continuous and $G(t, s) \leq \frac{1}{\Gamma(\delta)}$;

(3) for $0 \leq s<t \leq 1$, the $G(t, s)$ is monotone increasing with respect to $t$ and for $0 \leq t<s \leq 1$, the $G(t, s)$ is monotone decreasing with respect to $t$;

(4) $\frac{1}{\Gamma(\delta)}(\delta-1) t(1-t) s(1-s)^{\delta-1} \leq t^{2-\delta} G(t, s) \leq \frac{1}{\Gamma(\delta)} s(1-s)^{\delta-1}=G(s, s), t, s \in(0,1)$.

Proof (1) By the expression of $G(t, s)$, it is easy to see $G(t, s)>0, t, s \in(0,1)$.

(2) By the expression of $G(t, s)$, we can get results easily.

(3) According to the definition of $G(t, s)$, for $0<s<t \leq 1$, we have

$$
\begin{aligned}
\frac{\partial G(t, s)}{\partial t} & =\frac{\delta-1}{\Gamma(\delta)}\left(t^{\delta-2}(1-s)^{\delta-1}-(t-s)^{\delta-2}\right) \\
& \leq \frac{\delta-1}{\Gamma(\delta)}\left((t-s)^{\delta-2}(1-s)^{\delta-1}-(t-s)^{\delta-2}\right) \\
& \leq 0 .
\end{aligned}
$$

Therefore, $G(t, s)$ is monotone decreasing with respect to $t$ for $0 \leq s<t \leq 1$.

It is obvious $G(t, s)$ is monotone increasing with respect to $t$ for $0 \leq t<s \leq 1$.

(4) The result can be found in [13].

Lemma 2.8 If $u \in C_{2-\delta}^{0}[0,1], D_{0^{+}}^{\delta} u \in L_{1}[0,1]$, then the boundary value problem (1.1) and (1.2) is equivalent to the integral equation

$$
u(t)=\int_{0}^{1} G(t, s) f(s, u(s)) \mathrm{d} s+(b-a) t^{\delta-1}+a t^{\delta-2}, \quad t \in J .
$$

Proof By Lemma 2.6, we can easily see that Lemma 2.8 holds. 
Let

$$
P=\{u \in E: u(t) \geq 0, t \in J\}
$$

then $P$ is a cone in $E$.

For each $u \in P$, we have

$$
\lim _{t \rightarrow 0^{+}}\left[t^{2-\delta} \int_{0}^{1} G(t, s) f(s, u(s)) \mathrm{d} s+(b-a) t+a\right]=a .
$$

Let

$$
T u(t)=\int_{0}^{1} G(t, s) f(s, u(s)) \mathrm{d} s+(b-a) t^{\delta-1}+a t^{\delta-2} \quad \text { for } u \in P .
$$

Hence, $T: P \rightarrow E$.

Lemma 2.9 The operator $T: P \rightarrow P$ is completely continuous.

Proof It is easy to show $T: P \rightarrow P$.

(1) $T$ is a continuous operator.

If $\left\{u_{n}\right\} \subset P, u \in P$, and $\left\|u_{n}-u\right\| \rightarrow 0$ as $n \rightarrow \infty$, there exists a constant $\gamma>0$, such that $\left\|u_{n}\right\| \leq \gamma$ and $\|u\| \leq \gamma$, that is, for any $t \in J,\left|t^{2-\delta} u_{n}(t)\right| \leq \gamma,\left|t^{2-\delta} u(t)\right| \leq \gamma$.

Therefore, for a.e. $s \in J$, we can show

$$
\lim _{n \rightarrow \infty} f\left(s, u_{n}(s)\right)=\lim _{n \rightarrow \infty} f\left(s, s^{\delta-2} s^{2-\delta} u_{n}(s)\right)=f\left(s, s^{\delta-2} s^{2-\delta} u(s)\right)=f(s, u(s)),
$$

and there exists $\varphi_{\gamma} \in L_{1}[0,1]$, for a.e. $s \in J$,

$$
\left|f\left(s, u_{n}(s)\right)-f(s, u(s))\right| \leq 2 \varphi_{\gamma}(s) .
$$

Then by the Lebesgue dominated convergence theorem, we can get

$$
\begin{aligned}
\lim _{n \rightarrow \infty}\left\|T u_{n}-T u\right\| & =\lim _{n \rightarrow \infty} \sup _{t \in[0,1]}\left|t^{2-\delta} T u_{n}(t)-t^{2-\delta} T u(t)\right| \\
& \leq \lim _{n \rightarrow \infty} \sup _{t \in[0,1]} t^{2-\delta} \int_{0}^{1} G(t, s)\left|f\left(s, u_{n}(s)\right)-f(s, u(s))\right| \mathrm{d} s \\
& \leq \frac{1}{\Gamma(\delta)} \int_{0}^{1} \lim _{n \rightarrow \infty}\left|f\left(s, u_{n}(s)\right)-f(s, u(s))\right| \mathrm{d} s \\
& =0 .
\end{aligned}
$$

So $\lim _{n \rightarrow \infty}\left\|T u_{n}-T u\right\|=0$.

By Lemma 2.7, $a \geq 0$, and $b \geq 0$, we have $T u(t) \geq 0, t \in J$. Hence, $T: P \rightarrow P$ is continuous.

(2) $T: P \rightarrow P$ is relatively compact.

Let $A_{1}$ be any bounded set, then there exists a constant $\gamma>0$ such that $\|u\| \leq \gamma$ for each $u \in A_{1}$, that is, $\sup _{t \in J} t^{2-\delta}|u(t)| \leq \gamma$. 
There exists $\varphi_{\gamma} \in L_{1}[0,1]$ for any $u \in A_{1}$, we have

$$
\begin{aligned}
|f(s, u(s))| & =\left|f\left(s, s^{\delta-2} s^{2-\delta} u(s)\right)\right| \leq \varphi_{\gamma}(s), \quad s \in J \\
\left|t^{2-\delta} T u(t)\right| & =\mid \int_{0}^{1} t^{2-\delta} G(t, s) f(s, u(s) \mathrm{d} s+(b-a) t+a \mid \\
& \leq \frac{1}{\Gamma(\delta)} \int_{0}^{1} \varphi_{\gamma}(s) \mathrm{d} s+b+2 a
\end{aligned}
$$

It shows that $T\left(A_{1}\right)$ is uniformly bounded.

In addition, for any given $u \in A_{1}$, because the $G(t, s)$ is continuous for $(t, s) \in[0,1] \times$ $[0,1]$, then it must be uniformly continuous. So for any $\varepsilon>0$, there exists a constant $\sigma \in$ $\left(0, \frac{\varepsilon}{2(b+a)+1}\right)$ such that for any $t_{1}, t_{2}, s_{1}, s_{2} \in[0,1]$, as $\left|t_{1}-t_{2}\right|<\sigma$ and $\left|s_{1}-s_{2}\right|<\sigma$, we can get

$$
\left|t_{1}^{2-\delta} G\left(t_{1}, s_{1}\right)-t_{2}^{2-\delta} G\left(t_{2}, s_{2}\right)\right|<\frac{\varepsilon}{2 \int_{0}^{1} \varphi_{\gamma}(s) \mathrm{d} s+1}
$$

Then

$$
\begin{aligned}
& \left|t_{2}^{2-\delta} \operatorname{Tu}\left(t_{2}\right)-t_{1}^{2-\delta} \operatorname{Tu}\left(t_{1}\right)\right| \\
& \quad \leq \int_{0}^{1}\left|t_{2}^{2-\delta} G\left(t_{2}, s\right)-t_{1}^{2-\delta} G\left(t_{1}, s\right)\right||f(s, u(s))| \mathrm{d} s+|b-a|\left|t_{2}-t_{1}\right| \\
& \quad \leq \int_{0}^{1}\left|t_{2}^{2-\delta} G\left(t_{2}, s\right)-t_{1}^{2-\delta} G\left(t_{1}, s\right)\right|\left|\varphi_{\gamma}(s)\right| \mathrm{d} s+|b-a|\left|t_{2}-t_{1}\right| \\
& \quad<\frac{\varepsilon}{2 \int_{0}^{1} \varphi_{\gamma}(s) \mathrm{d} s+1} \int_{0}^{1} \varphi_{\gamma}(s) \mathrm{d} s+\frac{\varepsilon}{2(b+a)+1}(b+a) \\
& \quad=\varepsilon .
\end{aligned}
$$

Thus, we have proved $T\left(A_{1}\right)$ is equicontinuous.

By the Arzela-Ascoli theorem, we know that $T$ is relatively compact.

Therefore, $T$ is completely continuous.

\section{Comparison principle and the existence of solutions}

Definition 3.1 Let $\alpha \in E, D_{0^{+}}^{\delta} \alpha \in L_{1}[0,1]$, we say that $\alpha$ is a lower solution of the boundary value problem (1.1) and (1.2), if

$$
\begin{aligned}
& -D_{0^{+}}^{\delta} \alpha(t) \leq f(t, \alpha(t)), \quad \text { for a.e. } t \in(0,1) \\
& \lim _{t \rightarrow 0^{+}} t^{2-\delta} \alpha(t) \leq a, \quad \alpha(1) \leq b
\end{aligned}
$$

Let $\beta \in E, D_{0^{+}}^{\delta} \beta \in L_{1}[0,1]$, we say that $\beta$ is an upper solution of the boundary value problem (1.1) and (1.2), if

$$
\begin{aligned}
& -D_{0^{+}}^{\delta} \beta(t) \geq f(t, \beta(t)), \quad \text { for a.e. } t \in(0,1), \\
& \lim _{t \rightarrow 0^{+}} t^{2-\delta} \beta(t) \geq a, \quad \beta(1) \geq b .
\end{aligned}
$$


Lemma 3.1 If $u \in E, D_{0^{+}}^{\delta} u \in L_{1}[0,1]$ and it satisfies

$$
\begin{aligned}
& D_{0^{+}}^{\delta} u(t) \leq 0, \quad \text { for a.e. } t \in(0,1), \\
& \lim _{t \rightarrow 0^{+}} t^{2-\delta} u(t) \geq 0, \quad u(1) \geq 0,
\end{aligned}
$$

then $u(t) \geq 0$, for $t \in J$.

Proof Denote $-D_{0^{+}}^{\delta} u(t)=y(t)$, then $y(t) \geq 0$, for a.e. $t \in(0,1)$.

Let $\lim _{t \rightarrow 0^{+}} t^{2-\delta} u(t)=a, u(1)=b$, then $a \geq 0, b \geq 0$.

Now we consider the boundary value problem

$$
\begin{aligned}
& -D_{0^{+}}^{\delta} u(t)=y(t), \quad \text { for a.e. } t \in(0,1), \\
& \lim _{t \rightarrow 0^{+}} t^{2-\delta} u(t)=a, \quad u(1)=b .
\end{aligned}
$$

By Lemma 2.6, we have

$$
\begin{aligned}
u(t) & =\int_{0}^{1} G(t, s) y(s) \mathrm{d} s+(b-a) t^{\delta-1}+a t^{\delta-2} \\
& =\int_{0}^{1} G(t, s) y(s) \mathrm{d} s+a t^{\delta-2}(1-t)+b t^{\delta-1} .
\end{aligned}
$$

It follows that $u(t) \geq 0$ for $t \in J$ from Lemma 2.7.

Theorem 3.1 Suppose $(\mathrm{H})$ holds, there exist a nonnegative lower solution $\alpha$ and an upper solution $\beta$ of the boundary value problem (1.1) and (1.2) such that $\alpha(t) \leq \beta(t)$ for $t \in J$. Then the boundary value problem (1.1) and (1.2) has at least one positive solution $u$ and it satisfies $\alpha(t) \leq u(t) \leq \beta(t)$ for $t \in J$.

Proof Let

$$
f_{1}(t, u)= \begin{cases}f(t, \beta(t)), & u>\beta(t) \\ f(t, u), & \alpha(t) \leq u \leq \beta(t) \\ f(t, \alpha(t)), & u<\alpha(t)\end{cases}
$$

Since $f$ satisfies the $L_{1}$-Caratheódory conditions, we have $f_{1}$ satisfies $L_{1}$-Caratheódory conditions, too.

We consider the boundary value problem

$$
\begin{aligned}
& D_{0^{+}}^{\delta} u(t)+f_{1}(t, u(t))=0, \quad \text { for a.e. } t \in(0,1), \\
& \lim _{t \rightarrow 0^{+}} t^{2-\delta} u(t)=a, \quad u(1)=b .
\end{aligned}
$$

We define $T_{1}: P \rightarrow P$ by

$$
T_{1} u(t)=\int_{0}^{1} G(t, s) f_{1}(s, u(s)) \mathrm{d} s+(b-a) t^{\delta-1}+a t^{\delta-2} .
$$


By (H), for any $u \in P$ and $t \in J$, we have

$$
\begin{aligned}
0 & \leq f_{1}(t, u(t))=f_{1}\left(t, t^{\delta-2} t^{2-\delta} u(t)\right) \leq f_{1}\left(t, t^{\delta-2} t^{2-\delta} \beta(t)\right) \\
& \leq f_{1}\left(t, t^{\delta-2}\|\beta\|\right) \leq \varphi_{\|\beta\|}(t) .
\end{aligned}
$$

Define

$$
A_{2}=\left\{u \in P:\|u\| \leq \frac{1}{\Gamma(\delta)} \int_{0}^{1} \varphi_{\|\beta\|}(s) \mathrm{d} s+|b-a|+a\right\} .
$$

It is easy to see that $A_{2}$ is a closed and convex set.

By $(\mathrm{H})$, we can get

$$
\begin{aligned}
\left|t^{2-\delta} T_{1} u(t)\right| & =\left|\int_{0}^{1} t^{2-\delta} G(t, s) f_{1}(s, u(s)) \mathrm{d} s+(b-a) t+a\right| \\
& \leq \int_{0}^{1} G(t, s) f_{1}(s, u(s)) \mathrm{d} s+|b-a|+a \\
& \leq \int_{0}^{1} G(t, s) f\left(s, s^{\delta-2}\|\beta\|\right) \mathrm{d} s+|b-a|+a \\
& \leq \frac{1}{\Gamma(\delta)} \int_{0}^{1} \varphi_{\|\beta\|}(s) \mathrm{d} s+|b-a|+a .
\end{aligned}
$$

So

$$
\left\|T_{1} u\right\| \leq \frac{1}{\Gamma(\delta)} \int_{0}^{1} \varphi_{\|\beta\|}(s) \mathrm{d} s+|b-a|+a,
$$

that is, $T_{1}: A_{2} \rightarrow A_{2}$.

Similar to the proof of Lemma 2.9 , we can prove $T_{1}$ is completely continuous.

By the Schauder fixed point theorem, we can see that $T_{1}$ has at least one fixed point $u$, that is, there exists a positive solution $u$ of the boundary value problem (3.5) and (3.6).

Finally, we prove $\alpha(t) \leq u(t) \leq \beta(t)$, for $t \in J$.

Let $v(t)=u(t)-\alpha(t)$, by $(\mathrm{H})$, we have

$$
\begin{aligned}
& D_{0^{+}}^{\delta} \nu(t)=D_{0^{+}}^{\delta} u(t)-D_{0^{+}}^{\delta} \alpha(t) \\
&=-f_{1}(t, u(t))-D_{0^{+}}^{\delta} \alpha(t) \\
& \leq-f_{1}(t, u(t))+f_{1}(t, \alpha(t)) \\
& \leq 0, \\
& \lim _{t \rightarrow 0^{+}} t^{2-\delta} v(t)=\lim _{t \rightarrow 0^{+}} t^{2-\delta} u(t)-\lim _{t \rightarrow 0^{+}} t^{2-\delta} \alpha(t) \geq a-a=0,
\end{aligned}
$$

and

$$
v(1)=u(1)-\alpha(1) \geq b-b=0 .
$$

It follows that $v(t) \geq 0$ for $t \in J$ from Lemma 3.1.

Hence, we can show $u(t) \geq \alpha(t)$ for $t \in J$.

Similarly, we can get $u(t) \leq \beta(t)$ for $t \in J$. 
So each solution of the boundary value problem (3.5) and (3.6) satisfies $\alpha(t) \leq u(t) \leq \beta(t)$ for $t \in J$. That is, $f_{1}(t, u(t))=f(t, u(t))$ and $u$ is a positive solution of the boundary value problem (1.1) and (1.2).

\section{Impact of disturbance parameters $a, b$ on the existence of solutions}

Theorem 4.1 Let $(\mathrm{H})$ hold.

(1) If there exist parameters $a=\bar{a} \geq 0$ and $b=\bar{b} \geq 0$ such that the boundary value problem (1.1) and (1.2) has a positive solution $\bar{u}$, then for any $0 \leq a \leq \bar{a}$ and $0 \leq b \leq \bar{b}$, the boundary value problem (1.1) and (1.2) has a positive solution $u$ and $(b-a) t^{\delta-1}+a t^{\delta-2} \leq u \leq \bar{u}$ for $t \in J$.

(2) If there exist parameters $a=\bar{a} \geq 0$ and $b=\bar{b} \geq 0$ such that the boundary value problem (1.1) and (1.2) does not have positive solutions, then for any $a \geq \bar{a}$ and $b \geq \bar{b}$, the boundary value problem (1.1) and (1.2) does not have positive solutions.

Proof (1) Since there exist parameters $a=\bar{a} \geq 0$ and $b=\bar{b} \geq 0$ such that the boundary value problem (1.1) and (1.2) has a positive solution $\bar{u}$, by Lemma 2.8 , we have

$$
\bar{u}(t)=\int_{0}^{1} G(t, s) f(s, \bar{u}(s)) \mathrm{d} s+(\bar{b}-\bar{a}) t^{\delta-1}+\bar{a} t^{\delta-2} .
$$

Hence, for any $0 \leq a \leq \bar{a}$ and $0 \leq b \leq \bar{b}$,

$$
\bar{u}(t) \geq(\bar{b}-\bar{a}) t^{\delta-1}+\bar{a} t^{\delta-2} \geq(b-a) t^{\delta-1}+a t^{\delta-2}, \quad t \in J .
$$

We take $\alpha=(b-a) t^{\delta-1}+a t^{\delta-2}$ and $\beta=\bar{u}$, then

$$
\begin{aligned}
& -D_{0^{+}}^{\delta} \alpha(t)=-D_{0^{+}}^{\delta}\left((b-a) t^{\delta-1}+a t^{\delta-2}\right) \leq f(t, \alpha(t)), \quad t \in(0,1), \\
& \lim _{t \rightarrow 0^{+}} t^{2-\delta} \alpha(t)=\lim _{t \rightarrow 0^{+}} t^{2-\delta}\left((b-a) t^{\delta-1}+a t^{\delta-2}\right)=a, \quad \alpha(1)=b .
\end{aligned}
$$

We see that $\alpha$ and $\beta$ satisfy (3.1), (3.2), (3.3), and (3.4).

Therefore, $\alpha$ and $\beta$ are the lower solution and the upper solution of the boundary value problem (1.1) and (1.2), respectively.

For the parameters $0 \leq a \leq \bar{a}$ and $0 \leq b \leq \bar{b}$, by Theorem 3.1, we see that the boundary value problem (1.1) and (1.2) has at least one positive solution $u$ and $\alpha(t) \leq u(t) \leq \beta(t)$ for $t \in J$.

(2) If there exist constants $a_{0} \geq \bar{a}$ and $b_{0} \geq \bar{b}$ such that the boundary value problem (1.1) and (1.2) has a positive solution for the parameters $a_{0}$ and $b_{0}$, by (1), we can show that for each $0 \leq a \leq a_{0}$ and $0 \leq b \leq b_{0}$, the boundary value problem (1.1) and (1.2) has a positive solution. Therefore, the boundary value problem (1.1) and (1.2) has a positive solution for the parameters $\bar{a}$ and $\bar{b}$, which is a contradiction.

For convenience, we denote

$$
\begin{aligned}
& N=\frac{\Gamma(\delta+2)}{\Gamma(\delta+2)+1}, \quad M=\frac{2,048 \Gamma(\delta-1)}{11(\delta-1)}, \\
& f^{0}=\limsup _{u \rightarrow 0^{+}} \sup _{t \in J} \frac{f\left(t, t^{\delta-2} u\right)}{u}, \quad f_{\infty}=\liminf _{u \rightarrow+\infty} \inf _{t \in\left[\frac{1}{4}, \frac{3}{4}\right]} \frac{f(t, u)}{u} .
\end{aligned}
$$


Let

$$
\hat{P}=\left\{u \in E: t^{2-\delta} u(t) \geq(\delta-1) t(1-t)\|u\|, t \in J\right\} .
$$

Then $\hat{P}$ is a cone in $E$ and $\hat{P} \subset P$.

Lemma 4.1 (See [29]) Let E be a Banach space and $\hat{P} \subseteq E$ be a cone in E. Assume that $\Omega$ is a bounded open subset of $E$ and $\theta \in \Omega$ and that $T: \hat{P} \cap \bar{\Omega} \rightarrow \hat{P}$ is compact. If

$u \neq \tau T u \quad$ for all $u \in \hat{P} \cap \partial \Omega$ and $\tau \in[0,1]$

then the fixed point index $i(T, \hat{P} \cap \Omega, \hat{P})=1$.

Lemma 4.2 (See [29]) Let E be a Banach space and $\hat{P} \subseteq E$ be a cone in E. Assume that $\Omega$ is a bounded open subset of $E$ and that $T: \hat{P} \cap \bar{\Omega} \rightarrow \hat{P}$ is compact. If there exists $u_{0} \in \hat{P} \backslash\{\theta\}$ such that

$$
u-T u \neq \tau u_{0} \quad \text { for all } u \in \hat{P} \cap \partial \Omega \text { and } \tau \geq 0,
$$

then the fixed point index $i(T, \hat{P} \cap \Omega, \hat{P})=0$.

Lemma 4.3 If $u$ is a positive solution of the boundary value problem (1.1) and (1.2), then for $t \in J$,

$$
t^{2-\delta} u(t) \geq(\delta-1) t(1-t)\|u\| .
$$

Proof By Lemma 2.8, we can show

$$
u(t)=\int_{0}^{1} G(t, s) f(s, u(s)) \mathrm{d} s+(b-a) t^{\delta-1}+a t^{\delta-2} .
$$

It follows from Lemma 2.7, for $t \in J$, that

$$
\begin{aligned}
t^{2-\delta} u(t) & =\int_{0}^{1} t^{2-\delta} G(t, s) f(s, u(s)) \mathrm{d} s+(b-a) t+a \\
& \geq \int_{0}^{1} \frac{1}{\Gamma(\delta)}(\delta-1) t(1-t) s(1-s)^{\delta-1} f(s, u(s)) \mathrm{d} s+(1-t) a+b t \\
& \geq(\delta-1) t(1-t)\left(\frac{1}{\Gamma(\delta)} \int_{0}^{1} s(1-s)^{\delta-1} f(s, u(s)) \mathrm{d} s+b+a\right) .
\end{aligned}
$$

On the other hand,

$$
t^{2-\delta} u(t) \leq \frac{1}{\Gamma(\delta)} \int_{0}^{1} s(1-s)^{\delta-1} f(s, u(s)) \mathrm{d} s+b+a,
$$

we have

$$
\|u\| \leq \frac{1}{\Gamma(\delta)} \int_{0}^{1} s(1-s)^{\delta-1} f(s, u(s)) \mathrm{d} s+b+a .
$$


Hence,

$$
t^{2-\delta} u(t) \geq(\delta-1) t(1-t)\|u\|
$$

Theorem 4.2 Let $(\mathrm{H})$ hold, $f^{0}=0$, and $f_{\infty}=+\infty$.

(1) If $a=0$, the boundary value problem (1.1) and (1.2) has at least one positive solution for small enough $b$; the boundary value problem (1.1) and (1.2) has no positive solution for large enough $b$.

(2) If $b=0$, the boundary value problem (1.1) and (1.2) has at least one positive solution for small enough $a$; the boundary value problem (1.1) and (1.2) has no positive solution for large enough $a$.

(3) If $b=k a($ for any $k>0)$, the boundary value problem (1.1) and (1.2) has at least one positive solution for small enough $a$ and $b$; the boundary value problem (1.1) and

(1.2) has no positive solution for large enough $a$ and $b$.

Proof (1) By $f^{0}=0$, for given $N>0$, there exists a constant $r_{1}>0$ such that

$$
f\left(t, t^{\delta-2} u\right) \leq N u \leq N r_{1}, \quad \text { for } t \in J \text { and } u \in\left(0, r_{1}\right]
$$

Let

$$
\Omega_{1}=\left\{u \in E:\|u\|<r_{1}\right\}
$$

When $b<N r_{1}$, for any $u \in \hat{P} \cap \partial \Omega_{1}$, we have $\left|t^{2-\delta} u(t)\right| \leq r_{1}, t \in J$. By Lemma 2.7, we can get

$$
\begin{aligned}
t^{2-\delta}(T u)(t) & =\int_{0}^{1} t^{2-\delta} G(t, s) f(s, u(s)) \mathrm{d} s+(b-a) t+a \\
& =\int_{0}^{1} t^{2-\delta} G(t, s) f(s, u(s)) \mathrm{d} s+b t \\
& \leq \frac{1}{\Gamma(\delta)} \int_{0}^{1} s(1-s)^{\delta-1} f\left(s, s^{\delta-2} s^{2-\delta} u(s)\right) \mathrm{d} s+b \\
& <\frac{N r_{1}}{\Gamma(\delta)} \cdot \frac{\Gamma(\delta)}{\Gamma(\delta+1)}+N r_{1} \\
& =r_{1} \\
& =\|u\|,
\end{aligned}
$$

that is,

$$
\|T u\|<\|u\|, \quad u \in \hat{P} \cap \partial \Omega_{1} .
$$

We can show that

$$
u \neq \tau T u \quad \text { for all } u \in \hat{P} \cap \partial \Omega_{1} \text { and } \tau \in[0,1]
$$


Otherwise, there exist $u^{*} \in \hat{P} \cap \partial \Omega_{1}$ and $\tau^{*} \in[0,1]$ such that $u^{*}=\tau^{*} T u^{*}$, then

$$
\left\|u^{*}\right\|=\tau\left\|T u^{*}\right\| \leq\left\|T u^{*}\right\|
$$

which contradicts to (4.1).

In view of Lemma 4.1, we can get

$$
i\left(T, \hat{P} \cap \Omega_{1}, \hat{P}\right)=1
$$

On the other hand, by $f_{\infty}=+\infty$, for given $M>0$, there exists a constant $R>0$ such that

$$
f(t, u)>M u, \quad \text { for } t \in\left[\frac{1}{4}, \frac{3}{4}\right] \text { and } u \in[R,+\infty)
$$

Let $R_{1}>\frac{16}{3(\delta-1)} R$ and

$$
\Omega_{2}=\left\{u \in E:\|u\|<R_{1}\right\} .
$$

For any $u \in \hat{P} \cap \partial \Omega_{2}$, we have $u \in \hat{P}$ and $\|u\|=R_{1}$, so

$$
\min _{t \in\left[\frac{1}{4}, \frac{3}{4}\right]} u(t) \geq \min _{t \in\left[\frac{1}{4}, \frac{3}{4}\right]} t^{2-\delta} u(t) \geq \frac{3}{16}(\delta-1)\|u\|=\frac{3(\delta-1)}{16} R_{1}>R .
$$

By Lemma 2.7, we can get

$$
\begin{aligned}
t^{2-\delta}(T u)(t) & =\int_{0}^{1} t^{2-\delta} G(t, s) f(s, u(s)) \mathrm{d} s+(b-a) t+a \\
& =\int_{0}^{1} t^{2-\delta} G(t, s) f(s, u(s)) \mathrm{d} s+b t \\
& \geq \frac{1}{\Gamma(\delta)} \int_{0}^{1}(\delta-1) t(1-t) s(1-s)^{\delta-1} f(s, u(s)) \mathrm{d} s+b t
\end{aligned}
$$

When $t=\frac{1}{2}$, by (4.3) and (4.4), we can show

$$
\begin{aligned}
\left(\frac{1}{2}\right)^{2-\delta}(T u)\left(\frac{1}{2}\right) & >\frac{1}{4 \Gamma(\delta)} \int_{\frac{1}{4}}^{\frac{3}{4}}(\delta-1) s(1-s)^{\delta-1} M u(s) \mathrm{d} s \\
& \geq\left(\frac{3 M(\delta-1)^{2}}{64 \Gamma(\delta)} \int_{\frac{1}{4}}^{\frac{3}{4}} s(1-s)^{\delta-1} \mathrm{~d} s\right)\|u\| \\
& \geq\left(\frac{3 M(\delta-1)^{2}}{64 \Gamma(\delta)} \int_{\frac{1}{4}}^{\frac{3}{4}} s(1-s) \mathrm{d} s\right)\|u\| \\
& =\frac{11 M(\delta-1)}{2,048 \Gamma(\delta-1)}\|u\| \\
& =\|u\| .
\end{aligned}
$$

Hence,

$$
\|T u\|>\|u\|, \quad u \in \hat{P} \cap \partial \Omega_{2} .
$$


In the following, we show that there exists $u_{0} \in \hat{P} \backslash\{\theta\}$ such that

$$
u-T u \neq \tau T u_{0} \quad \text { for all } u \in \hat{P} \cap \partial \Omega_{2} \text { and } \tau \geq 0 .
$$

If this is not true, then there exist $u^{*} \in \hat{P} \cap \partial \Omega_{2}$ and $\tau^{*} \geq 0$ such that $u^{*}-T u^{*}=\tau^{*} u_{0}$, so

$$
u^{*}=T u^{*}+\tau^{*} u_{0} \geq T u^{*}
$$

and

$$
\left\|u^{*}\right\| \geq\left\|T u^{*}\right\|
$$

which contradicts (4.5).

By Lemma 4.2, we have

$$
i\left(T, \hat{P} \cap \Omega_{2}, \hat{P}\right)=0 .
$$

By (4.2), (4.6), and the additivity property of the fixed point index, we obtain

$$
i\left(T, \hat{P} \cap\left(\Omega_{2} \backslash \bar{\Omega}_{1}\right), \hat{P}\right)=i\left(T, \hat{P} \cap \Omega_{2}, \hat{P}\right)-i\left(T, \hat{P} \cap \overline{\Omega_{1}}, \hat{P}\right)=0-1=-1 \neq 0 .
$$

Then $T$ has at least one fixed point $u \in \hat{P} \cap\left(\Omega_{2} \backslash \bar{\Omega}_{1}\right)$, which implies the boundary value problem (1.1) and (1.2) has at least one positive solution.

In the following, we prove that there exists large enough $b$ such that the boundary value problem (1.1) and (1.2) has no positive solution.

Otherwise, for any large enough $b_{n}$, the boundary value problem (1.1) and (1.2) has solutions, then there exists $\left\{b_{n}\right\}$ such that, for $\lim _{n \rightarrow+\infty} b_{n}=+\infty$, the boundary value problem

$$
\begin{aligned}
& D_{0^{+}}^{\delta} u(t)+f(t, u(t))=0, \quad \text { for a.e. } t \in(0,1), \\
& \lim _{t \rightarrow 0^{+}} t^{2-\delta} u(t)=0, \quad u(1)=b_{n}
\end{aligned}
$$

has a positive solution, which we denote $u_{n}$.

Due to this,

$$
\begin{aligned}
u_{n}(1) & =\int_{0}^{1} G(1, s) f\left(s, u_{n}(s)\right) \mathrm{d} s+b_{n} \\
& \geq b_{n} \rightarrow+\infty \quad(n \rightarrow+\infty),
\end{aligned}
$$

so $\left\|u_{n}\right\| \rightarrow+\infty$.

In addition, by $f_{\infty}=+\infty$ and given $M>0$, there exists a constant $\bar{R}>0$ such that $f(t, u)>$ $2 M u, t \in\left[\frac{1}{4}, \frac{3}{4}\right]$, and $u \in\left[\bar{R}_{1},+\infty\right)$.

Let $\bar{R}_{1}>\frac{16}{3(\delta-1)} \bar{R}$. For any $u \in \partial \hat{P}_{\bar{R}_{1}}=\left\{u \mid u \in \hat{P},\|u\|=\bar{R}_{1}\right\}$, we have $\left|t^{2-\delta} u\right| \leq \bar{R}_{1}$, so

$$
\min _{t \in\left[\frac{1}{4}, \frac{3}{4}\right]} u(t) \geq \min _{t \in\left[\frac{1}{4}, \frac{3}{4}\right]} t^{2-\delta} u(t) \geq \frac{3}{16}(\delta-1)\|u\|=\frac{3(\delta-1)}{16} \bar{R}_{1}>\bar{R} .
$$


Similar to the proof above, we can see that, for large integer $n$,

$$
\left(\frac{1}{2}\right)^{2-\delta} u_{n}\left(\frac{1}{2}\right)>2 \cdot \frac{11 M(\delta-1)}{2,048 \Gamma(\delta-1)}\left\|u_{n}\right\|=2\left\|u_{n}\right\| .
$$

So $\left\|u_{n}\right\|>2\left\|u_{n}\right\|$, which is a contradiction.

(2) Similar to the proof of (1), we can prove (2) holds.

(3) By $f^{0}=0$ and given $N>0$, there exists $\tilde{r}_{1}>0$ such that

$$
f\left(t, t^{\delta-2} u\right) \leq N u<N \tilde{r}_{1}, \quad \text { for } t \in J \text { and } u \in\left(0, \tilde{r}_{1}\right] .
$$

Let

$$
\Omega_{3}=\left\{u \in E:\|u\|<\tilde{r}_{1}\right\} .
$$

Since $b=k a$ and $k>0$, let $\max \{a, b\}=a \max \{1, k\}<N \tilde{r}_{1}$, we have $0 \leq a((k-1) t+1) \leq$ $a \max \{1, k\}<N \tilde{r}_{1}$.

Hence, for any $u \in \hat{P} \cap \partial \Omega_{3}$, by Lemma 2.7, we can get

$$
\begin{aligned}
t^{2-\delta}(T u) t & =\int_{0}^{1} t^{2-\delta} G(t, s) f(s, u(s)) \mathrm{d} s+(b-a) t+a \\
& =\int_{0}^{1} t^{2-\delta} G(t, s) f(s, u(s)) \mathrm{d} s+(k a-a) t+a \\
& \leq \frac{1}{\Gamma(\delta)} \int_{0}^{1} s(1-s)^{\delta-1} f\left(s, s^{\delta-2} s^{2-\delta} u(s)\right) \mathrm{d} s+a((k-1) t+1) \\
& <N\left(\frac{1}{\Gamma(\delta+2)}+1\right) \tilde{r}_{1} \\
& =\tilde{r}_{1} \\
& =\|u\|
\end{aligned}
$$

and

$$
\lim _{t \rightarrow 0^{+}} t^{2-\delta}(T u) t=a<N \tilde{r}_{1}<\tilde{r}_{1} .
$$

This implies $\|T u\|<\|u\|$ for $u \in \hat{P} \cap \partial \Omega_{3}$.

It is similar to the proof of (1), by Lemma 4.1, we have

$$
i\left(T, \hat{P} \cap \Omega_{3}, \hat{P}\right)=1 \text {. }
$$

On the other hand, by $f_{\infty}=+\infty$ and given $M>0$, similar to the proof of (1), we can see that there exists a constant $\tilde{R}_{1}$ such that

$$
\Omega_{4}=\left\{u \in E:\|u\|<\tilde{R}_{1}\right\}
$$

and

$$
i\left(T, \hat{P} \cap \Omega_{4}, \hat{P}\right)=0 .
$$


By (4.8) and (4.9), and the additivity property of the fixed point index, we obtain

$$
i\left(T, \hat{P} \cap\left(\Omega_{4} \backslash \bar{\Omega}_{3}\right), \hat{P}\right)=i\left(T, \hat{P} \cap \Omega_{4}, \hat{P}\right)-i\left(T, \hat{P} \cap \bar{\Omega}_{3}, \hat{P}\right)=0-1=-1 \neq 0,
$$

then $T$ has at least one fixed point $u \in \Omega_{4} \backslash \bar{\Omega}_{3}$, that is, the boundary value problem (1.1) and (1.2) has a positive solution.

Similar to the proof of (1), we can prove that the boundary value problem (1.1) and (1.2) has no positive solution for large enough $a$ and $b$.

In summary, the boundary value problem (1.1) and (1.2) has at least one positive solution for small enough $a$ and $b$; the boundary value problem (1.1) and (1.2) has no solution for large enough $a$ and $b$.

Theorem 4.3 Let $f_{\infty}=+\infty$ and $\Lambda \subset \mathbb{R}_{+}^{2}$ be a bounded set. Then for each parameters $(a, b) \in \Lambda$, there exists a constant $\eta(\Lambda)$ such that the positive solution $u$ of the boundary value problem (1.1) and (1.2) satisfies $\|u\| \leq \eta(\Lambda)$.

Proof Because $\Lambda$ is a bounded set, there are constants $\eta_{1}, \eta_{2}$, such that for each parameter $(a, b) \in \Lambda, 0 \leq a \leq \eta_{1}, 0 \leq b \leq \eta_{2}$.

Since $f_{\infty}=+\infty$, for $M>0$, there exists a constant $R>0$ such that

$$
f(t, u)>M u, \quad u \in[R,+\infty), t \in J .
$$

Let $\eta=\frac{16 R}{3(\delta-1)}$, we can show $\|u\| \leq \eta$.

Otherwise, $\|u\|>\eta$. Hence,

$$
\min _{t \in\left[\frac{1}{4}, \frac{3}{4}\right]} u(t) \geq \min _{t \in\left[\frac{1}{4}, \frac{3}{4}\right]} t^{2-\delta} u(t) \geq \frac{3(\delta-1)}{16}\|u\|>\frac{3(\delta-1)}{16} \eta=R .
$$

By Lemma 2.7, we have

$$
\begin{aligned}
t^{2-\delta} u(t) & =\int_{0}^{1} t^{2-\delta} G(t, s) f(s, u(s)) \mathrm{d} s+(b-a) t+a \\
& \geq \frac{1}{\Gamma(\delta)} \int_{\frac{1}{4}}^{\frac{3}{4}}(\delta-1) t(1-t) s(1-s)^{\delta-1} f(s, u(s)) \mathrm{d} s+(b-a) t+a .
\end{aligned}
$$

When $t=\frac{1}{2}$, we can show

$$
\begin{aligned}
\left(\frac{1}{2}\right)^{2-\delta} u\left(\frac{1}{2}\right) & >\frac{1}{4 \Gamma(\delta)} \int_{\frac{1}{4}}^{\frac{3}{4}}(\delta-1) s(1-s)^{\delta-1} M u(s) \mathrm{d} s \\
& \geq \frac{M(\delta-1)}{4 \Gamma(\delta)} \int_{\frac{1}{4}}^{\frac{3}{4}} s(1-s)^{\delta-1} s^{2-\delta} u(s) \mathrm{d} s \\
& \geq\left(\frac{3 M(\delta-1)^{2}}{64 \Gamma(\delta)} \int_{\frac{1}{4}}^{\frac{3}{4}} s(1-s)^{\delta-1} \mathrm{~d} s\right)\|u\| \\
& \geq\left(\frac{3 M(\delta-1)^{2}}{64 \Gamma(\delta)} \int_{\frac{1}{4}}^{\frac{3}{4}} s(1-s) \mathrm{d} s\right)\|u\|
\end{aligned}
$$




$$
\begin{aligned}
& =\frac{11 M(\delta-1)}{2,048 \Gamma(\delta-1)}\|u\| \\
& =\|u\|,
\end{aligned}
$$

which is a contradiction.

Theorem 4.4 Let $(\mathrm{H})$ hold. If the $f^{0}=0$ and $f_{\infty}=+\infty$, then there exists a continuous curve $L \subseteq \mathbb{R}_{+}^{2}=[0,+\infty) \times[0,+\infty)$ such that the following conclusions hold:

(1) $\mathbb{R}_{+}^{2}$ is divided into two separate sub-regions by $L: \Lambda^{E}, \Lambda^{N}$, and $L \subseteq \Lambda^{E}$.

(2) The boundary value problem (1.1) and (1.2) has at least two positive solutions for each parameters $(a, b) \in \Lambda^{E} \backslash L$; the boundary value problem (1.1) and (1.2) has at least one positive solution for $(a, b) \in L$; the boundary value problem (1.1) and (1.2) does not have a positive solution for any $(a, b) \in \Lambda^{N}$.

Proof Let

$$
\Lambda^{E}=\left\{(a, b) \in \mathbb{R}_{+}^{2}:\right.
$$

the boundary value problem (1.1) and (1.2) has at least one positive solution\}.

By Theorem 4.2, $\Lambda^{E} \neq \varnothing$. For any $(\tilde{a}, \tilde{b}) \in \Lambda^{E}$, we define

$$
S(\tilde{a}, \tilde{b})=\left\{(a, b) \in \mathbb{R}_{+}^{2}: 0 \leq a \leq \tilde{a}, 0 \leq b \leq \tilde{b}\right\} .
$$

By Theorem 4.1, $S \subseteq \Lambda^{E}$ if and only if $(\tilde{a}, \tilde{b}) \in \Lambda^{E}$. So

$$
\Lambda^{E}=\bigcup_{(\tilde{a}, \tilde{b}) \in \Lambda^{E}} S .
$$

Let $\Lambda^{N}=\mathbb{R}_{+}^{2} \backslash \Lambda^{E}$, by Theorem $4.2, \Lambda^{N} \neq \varnothing$, and $\Lambda^{E} \cup \Lambda^{N}=\mathbb{R}_{+}^{2}$.

(i) It follows from Theorem 4.2 that the $\Lambda^{E}$ is a bounded set.

(ii) Let $L$ be the boundary of $\Lambda^{E}$. We can prove that the boundary value problem (1.1) and (1.2) has at least one positive solution for $(a, b) \in L$. That is $L \subseteq \Lambda^{E}$.

Take $(a, b) \in L$, then there exists a sequence $\left\{\left(a_{m}, b_{m}\right)\right\}_{m=1}^{\infty} \subseteq \Lambda^{E}$, such that $a_{m} \rightarrow a, b_{m} \rightarrow$ $b,(m \rightarrow \infty)$. Let $u_{m}(t)$ be the solution of the boundary value problem (1.1) and (1.2) for the parameters $a=a_{m}$ and $b=b_{m}$. By Theorem 4.3, there exists a constant $\eta=\eta\left(\Lambda^{E}\right)$ such that $\left\|u_{m}\right\| \leq \eta$. Then $\left\{u_{m}(t)\right\}_{m=1}^{\infty}$ is uniformly bounded and we can prove $\left\{u_{m}(t)\right\}_{m=1}^{\infty}$ is equicontinuous, similar to the proof of Lemma 2.9.

It follows that $\left\{u_{m}(t)\right\}_{m=1}^{\infty}$ has a subsequence which is uniformly convergent on $J$ from the Ascoli-Arzela theorem. Without loss of generality, we can assume that $\left\{u_{m}(t)\right\}_{m=1}^{\infty}$ itself converges uniformly to $u$ on $J$, then $u \in P$. Since $f$ satisfies the $L_{1}$-Caratheódory conditions and

$$
u_{m}(t)=\int_{0}^{1} G(t, s) f\left(s, u_{m}(s)\right) \mathrm{d} s+\left(b_{m}-a_{m}\right) t^{\delta-1}+a_{m} t^{\delta-2},
$$

letting $m \rightarrow \infty$, by the Lebesgue dominated convergence theorem, we can get

$$
u(t)=\int_{0}^{1} G(t, s) f(s, u(s)) \mathrm{d} s+(b-a) t^{\delta-1}+a t^{\delta-2} .
$$


So the boundary value problem (1.1) and (1.2) has at least one positive solution for the parameters $(a, b) \in L$ and $L \subseteq \Lambda^{E}$. By Lemma 4.3, we have $u \in \hat{P}$.

(iii) By Theorem 4.1, the boundary value problem (1.1) and (1.2) has at least one positive solution for $(a, b) \in \Lambda^{E}$, has no positive solution for any $(a, b) \in \Lambda^{N}$, and $L$ is the boundary of $\Lambda^{E}$.

(iv) We can prove that the boundary value problem (1.1) and (1.2) has at least two positive solutions for $(a, b) \in \Lambda^{E} \backslash L$.

Let $(a, b)=\left(a_{0}, b_{0}\right) \in \Lambda^{E} \backslash L$ be fixed, then there exist $(\bar{a}, \bar{b})$ and $(\underline{a}, \underline{b}) \in \Lambda^{E} \backslash L$ such that $\underline{a}<a_{0}<\bar{a}$ and $\underline{b}<b_{0}<\bar{b}$, the boundary value problem (1.1) and (1.2) has a solution $\bar{u}$ for $(a, b)=(\bar{a}, \bar{b})$. In view of Theorem 4.1, the boundary value problem (1.1) and (1.2) has at least one positive solution $u_{1}$, and $u_{1} \leq \bar{u}$ for $(a, b)=\left(a_{0}, b_{0}\right)$. Similarly, the boundary value problem (1.1) and (1.2) has a solution $\underline{u}$ for $(a, b)=(\underline{a}, \underline{b})$ and $\underline{u} \leq u_{1}$.

Hence, $\underline{u}<u_{1}<\bar{u}$.

Let $\alpha=\underline{u}, \beta=\bar{u}$, Obviously, $\alpha, \beta$ are the lower solution and the upper solution of the boundary value problem (1.1) and (1.2) for $(a, b)=\left(a_{0}, b_{0}\right)$, respectively.

Let $(\hat{a}, \hat{b}) \in \Lambda^{N}$ such that $a_{0}<\hat{a}$ and $b_{0}<\hat{b}$.

Define $K:\left[a_{0}, \hat{a}\right] \times\left[b_{0}, \hat{b}\right] \times \hat{P} \rightarrow \hat{P}$,

$$
K\left(k_{1}, k_{2}, u\right)=\int_{0}^{1} G(t, s) f(s, u(s)) \mathrm{d} s+\left(k_{2}-k_{1}\right) t^{\delta-1}+k_{1} t^{\delta-2} .
$$

Let

$$
F(t, u)= \begin{cases}f(t, \beta(t)), & u>\beta(t) \\ f(t, u), & \alpha(t) \leq u \leq \beta(t) \\ f(t, \alpha(t)), & u<\alpha(t)\end{cases}
$$

define $\hat{K}:\left[a_{0}, \hat{a}\right] \times\left[b_{0}, \hat{b}\right] \times \hat{P} \rightarrow \hat{P}$,

$$
\hat{K}\left(k_{1}, k_{2}, u\right)=\int_{0}^{1} G(t, s) F(s, u(s)) \mathrm{d} s+\left(k_{2}-k_{1}\right) t^{\delta-1}+k_{1} t^{\delta-2} .
$$

Similar to the proof of Lemma 2.9, for each $\left(k_{1}, k_{2}\right) \in\left[a_{0}, \hat{a}\right] \times\left[b_{0}, \hat{b}\right]$, we can show $K$ and $\hat{K}$ are completely continuous operators.

By Lemma 2.8, $u$ is a positive solution of the boundary value problem (1.1) and (1.2) if and only if $u=K(a, b, u)$.

Define a linear segmentation

$$
S_{0}=\left\{\left(k_{1}, k_{2}\right) \in \mathbb{R}_{+}^{2}: k_{1}=(1-\tau) a_{0}+\tau \hat{a}, k_{2}=(1-\tau) b_{0}+\tau \hat{b}, \tau \in[0,1]\right\} .
$$

Let $\Lambda \subseteq \mathbb{R}_{+}^{2}$ be a bounded set such that $S_{0} \cap \Lambda^{E} \subseteq \Lambda,(\underline{a}, \underline{b}) \in \Lambda,(\bar{a}, \bar{b}) \in \Lambda$, and $\eta=\eta(\Lambda)$ given by Theorem 4.3.

Let

$$
\Omega=\{u \in \hat{P}:\|u\|<\eta, \alpha(t)<u(t)<\beta(t), t \in J\},
$$

then $\Omega$ is a nonempty bounded open set of $\hat{P}$ and $u_{1} \in \Omega$. 
Since $F$ is bounded, there exists a constant $R>\eta>0$ such that for each $\left(k_{1}, k_{2}, u\right) \in$ $\left[a_{0}, \hat{a}\right] \times\left[b_{0}, \hat{b}\right] \times \hat{P}$, we have $\left|t^{2-\delta} \hat{K}\left(k_{1}, k_{2}, u\right)\right|<R$.

Let

$$
B(\theta, R)=\{u \in E:\|u\|<R\} .
$$

It is easy to show $\Omega \subset \hat{P} \cap B(\theta, R)$ and $u \neq \mu \hat{K} u$ when $u \in \hat{P} \cap \partial B(\theta, R)$ and $\mu \in[0,1]$.

By Lemma 4.1, for each $a \leq k_{1} \leq \hat{a}, b \leq k_{2} \leq \hat{b}$, we have

$$
i\left(\hat{K}\left(k_{1}, k_{2}, u\right), \hat{P} \cap B(\theta, R), \hat{P}\right)=1 .
$$

Due to $\hat{K}$ having no fixed point on $\hat{P} \cap(\overline{B(\theta, R)} \backslash \Omega)$, for each $a_{0} \leq k_{1} \leq \hat{a}, b_{0} \leq k_{2} \leq \hat{b}$,

$$
i\left(\hat{K}\left(k_{1}, k_{2}, u\right), \hat{P} \cap(B(\theta, R) \backslash \bar{\Omega}), \hat{P}\right)=0 .
$$

We notice $\left.\hat{K}\right|_{\Omega}=K$, by the excision property of the fixed point index, for each $a_{0} \leq k_{1} \leq$ $\hat{a}, b_{0} \leq k_{2} \leq \hat{b}$, we have

$$
\begin{aligned}
& i\left(K\left(k_{1}, k_{2}, u\right), \hat{P} \cap \Omega, \hat{P}\right) \\
& \quad=i\left(\hat{K}\left(k_{1}, k_{2}, u\right), \hat{P} \cap \Omega, \hat{P}\right) \\
& \quad=i\left(\hat{K}\left(k_{1}, k_{2}, u\right), \hat{P} \cap B(\theta, R), \hat{P}\right)-i\left(\hat{K}\left(k_{1}, k_{2}, u\right), \hat{P} \cap(B(\theta, R) \backslash \bar{\Omega}), \hat{P}\right) \\
& \quad=1 .
\end{aligned}
$$

Because $(\hat{a}, \hat{b}) \in \Lambda^{N}$, for each $u \in \hat{P}$, we have $K(\hat{a}, \hat{b}, u) \neq u$ and

$$
i(\hat{K}(\hat{a}, \hat{b}, u), \hat{P} \cap B(\theta, R), \hat{P})=0 .
$$

Define $H:[0,1] \times(\hat{P} \cap \overline{B(\theta, R)}) \rightarrow C_{2-\delta}^{0}[0,1]$,

$$
H(\tau, u)=K\left((1-\tau) a_{0}+\tau \hat{a},(1-\tau) b_{0}+\tau \hat{b}, u\right),
$$

then $H$ is completely continuous.

We can prove $H(\tau, u) \neq u$ for $(\tau, u) \in[0,1] \times(\hat{P} \cap \partial B(\theta, R))$.

Otherwise, there exists $\left(\tau_{0}, u_{0}\right) \in[0,1] \times(\hat{P} \cap \partial B(\theta, R))$ such that $H\left(\tau_{0}, u_{0}\right)=u_{0}$, that is

$$
K\left(\left(1-\tau_{0}\right) a_{0}+\tau_{0} \hat{a},\left(1-\tau_{0}\right) b_{0}+\tau_{0} \hat{b}, u_{0}\right)=u_{0}, \quad u_{0} \in \hat{P},\left\|u_{0}\right\|=R .
$$

So for $\tilde{k}_{1}=\left(1-\tau_{0}\right) a_{0}+\tau_{0} \hat{a}, \tilde{k}_{2}=\left(1-\tau_{0}\right) b_{0}+\tau_{0} \hat{b}$, and $\left(\tilde{k}_{1}, \tilde{k}_{2}\right) \in \Lambda, u_{0}$ is a solution of the boundary value problem (1.1) and (1.2), by Theorem $4.3,\left\|u_{0}\right\|<\eta$, which is a contradiction. According to homotopy invariance of the fixed point index and (4.10), it follows that

$$
\begin{aligned}
i\left(K\left(a_{0}, b_{0}, u\right), \hat{P} \cap B(\theta, R), \hat{P}\right) & =i(H(0, u), \hat{P} \cap B(\theta, R), \hat{P}) \\
& =i(H(1, u), \hat{P} \cap B(\theta, R), \hat{P}) \\
& =i(\hat{K}(\hat{a}, \hat{b}, u), \hat{P} \cap B(\theta, R), \hat{P}) \\
& =0 .
\end{aligned}
$$


By (4.11) and (4.12),

$$
i\left(K\left(a_{0}, b_{0}, u\right), \hat{P} \cap(B(\theta, R) \backslash \bar{\Omega}), \hat{P}\right)=-1 .
$$

Hence, the boundary value problem (1.1) and (1.2) has a solution $u_{2} \in \hat{P} \cap(B(\theta, R) \backslash \bar{\Omega})$ for $(a, b)=\left(a_{0}, b_{0}\right)$.

Since $u_{1} \in \Omega$, then $u_{1} \neq u_{2}$, that is, the boundary value problem (1.1) and (1.2) has at least two positive solutions for $(a, b)=\left(a_{0}, b_{0}\right)$.

\section{Illustration}

To illustrate our main results, we present the following examples.

Example 5.1 We consider the boundary value problem which is composed of the equation

$$
D_{0^{+}}^{\frac{3}{2}} u(t)+\mathrm{e}^{t^{\frac{q}{2}} u^{q}}-1=0, \quad \text { for a.e. } t \in(0,1),
$$

and nonhomogeneous boundary conditions

$$
\lim _{t \rightarrow 0^{+}} t^{\frac{1}{2}} u(t)=a, \quad u(1)=b,
$$

where $q>1$.

Let $f(t, u)=\mathrm{e}^{\mathrm{e}^{\frac{q}{2}} u^{q}}-1$. Clearly, $\delta=\frac{3}{2}$, it is easy to see that $(\mathrm{H})$ holds,

$$
f^{0}=\limsup _{u \rightarrow 0^{+}} \sup _{t \in J} \frac{f\left(t, t^{-\frac{1}{2}} u\right)}{u}=\limsup _{u \rightarrow 0^{+}} \frac{\mathrm{e}^{u^{q}}-1}{u}=0
$$

and

$$
f_{\infty}=\liminf _{u \rightarrow+\infty} \inf _{t \in\left[\frac{1}{4}, \frac{3}{4}\right]} \frac{f(t, u)}{u}=\liminf _{u \rightarrow+\infty} \frac{\mathrm{e}^{\left(\frac{u}{2}\right)^{q}}-1}{u}=+\infty .
$$

By Theorem 4.4, we see that there exists a bounded and continuous curve $L$ in $\mathbb{R}_{+}^{2}$ separating $\mathbb{R}_{+}^{2}$ into two disjoint subsets $\Lambda^{E}$ and $\Lambda^{N}$ with $L \subseteq \Lambda^{E}$ such that boundary value problem (5.1) and (5.2) has at least two positive solutions for each for each parameters $(a, b) \in \Lambda^{E} \backslash L$, one positive solution for $(a, b) \in L$ and no positive solution for any $(a, b) \in \Lambda^{N}$.

Example 5.2 We consider the boundary value problem which is composed of the equation

$$
D_{0^{+}}^{\frac{7}{4}} u(t)+(t \sin t+t) u^{2}+t^{\frac{3}{4}} u^{\frac{3}{2}} \arctan t u=0, \quad \text { for a.e. } t \in(0,1)
$$

and nonhomogeneous boundary conditions

$$
\lim _{t \rightarrow 0^{+}} t^{\frac{1}{4}} u(t)=a, \quad u(1)=b .
$$


Let $f(t, u)=t(\sin t+1) u^{2}+t^{\frac{3}{4}} u^{\frac{3}{2}} \arctan t u$. Clearly, $\delta=\frac{7}{4}$, it is easy to see that $(\mathrm{H})$ holds,

$$
f^{0}=\limsup _{u \rightarrow 0^{+}} \sup _{t \in J} \frac{f\left(t, t^{-\frac{1}{2}} u\right)}{u}=\limsup _{u \rightarrow 0^{+}}\left((\sin 1+1) u+u^{\frac{1}{2}} \arctan u\right)=0,
$$

and

$$
f_{\infty}=\liminf _{u \rightarrow+\infty} \inf _{t \in\left[\frac{1}{4}, \frac{3}{4}\right]} \frac{f(t, u)}{u}=\liminf _{u \rightarrow+\infty}\left(\left(\sin \frac{1}{4}+1\right) \frac{1}{4} u+\left(\frac{1}{4}\right)^{\frac{3}{4}} u^{\frac{1}{2}} \arctan \frac{1}{4} u\right)=+\infty
$$

By Theorem 4.4, we see that there exists a bounded and continuous curve $L$ in $\mathbb{R}_{+}^{2}$ separating $\mathbb{R}_{+}^{2}$ into two disjoint subsets $\Lambda^{E}$ and $\Lambda^{N}$ with $L \subseteq \Lambda^{E}$ such that the boundary value problem (5.3) and (5.4) has at least two positive solutions for each for each parameters $(a, b) \in \Lambda^{E} \backslash L$, one positive solution for $(a, b) \in L$ and no positive solution for any $(a, b) \in \Lambda^{N}$.

Example 5.3 We consider the boundary value problem which is composed of the equation

$$
D_{0^{+}}^{\frac{5}{3}} u(t)+(1+a(t)) t^{\frac{p}{2}} u^{p}=0, \quad \text { for a.e. } t \in(0,1)
$$

and nonhomogeneous boundary conditions

$$
\lim _{t \rightarrow 0^{+}} t^{\frac{1}{3}} u(t)=a, \quad u(1)=b,
$$

where $p>1, a(t)$ is a continuous function for $t \in[0,1]$ and $a(t)>0, t \in\left[\frac{1}{4}, \frac{3}{4}\right]$.

Let $f(t, u)=(1+a(t)) t^{\frac{p}{2}} u^{p}$. Clearly, $\delta=\frac{5}{3}$, it is easy to see that $(\mathrm{H})$ holds,

$$
f^{0}=\limsup _{u \rightarrow 0^{+}} \sup _{t \in J} \frac{f\left(t, t^{-\frac{1}{2}} u\right)}{u}=\sup _{t \in J}(1+a(t)) \limsup _{u \rightarrow 0^{+}} u^{p-1}=0
$$

and

$$
f_{\infty}=\liminf _{u \rightarrow+\infty} \inf _{t \in\left[\frac{1}{4}, \frac{3}{4}\right]} \frac{f(t, u)}{u}=\inf _{t \in\left[\frac{1}{4}, \frac{3}{4}\right]}(1+a(t)) t^{\frac{p}{2}} \liminf _{u \rightarrow+\infty} u^{p-1}=+\infty
$$

By Theorem 4.4, we see that there exists a bounded and continuous curve $L$ in $\mathbb{R}_{+}^{2}$ separating $\mathbb{R}_{+}^{2}$ into two disjoint subsets $\Lambda^{E}$ and $\Lambda^{N}$ with $L \subseteq \Lambda^{E}$ such that the boundary value problem (5.5) and (5.6) has at least two positive solutions for each for each parameters $(a, b) \in \Lambda^{E} \backslash L$, one positive solution for $(a, b) \in L$, and no positive solution for any $(a, b) \in \Lambda^{N}$.

\section{Conclusions}

The existence and nonexistence of positive solutions for fractional differential equations with nonhomogeneous boundary conditions is an important topic in the area of fractional calculus. When solving the actual problems with the boundary value problems of differential equations, the disturbance parameter under boundary conditions is a very important 
factor. Moreover, it is always inevitable that the impact of such a disturbance parameter to the existence of solutions always exists.

In this paper, by using the upper and lower solution method and fixed point theorem, we obtain the sufficient conditions for the boundary value problem to have at least one positive solution, two positive solutions, and no positive solution, respectively. Moreover, under certain conditions, we prove that there exists a bounded and continuous curve $L$ dividing $[0,+\infty) \times[0,+\infty)$ into two separate subsets $\Lambda^{E}$ and $\Lambda^{N}$ with $L \subseteq \Lambda^{E}$ such that the boundary value problem has at least two positive solutions for each $(a, b) \in \Lambda^{E} \backslash L$, one positive solution for each $(a, b) \in L$, and no positive solution for any $(a, b) \in \Lambda^{N}$.

\section{Competing interests}

The authors declare that they have no competing interests.

\section{Authors' contributions}

The authors declare that the work was realized in collaboration with the same responsibility. All authors read and approved the final manuscript.

\section{Acknowledgements}

The authors would like to thank the anonymous referee of this paper for very helpful comments and suggestions which improved final version of this paper.

This work is supported by the National Natural Science Foundation of China (No. 11171220) and the Hujiang Foundation of China (B14005).

Received: 10 September 2015 Accepted: 11 January 2016 Published online: 28 January 2016

\section{References}

1. Diethelm, K: The Analysis of Fractional Differential Equations. Lecture Notes in Mathematics, vol. 2004. Springer, Berlin (2010)

2. Podlubny, I: Fraction Differential Equations. Academic Press, New York (1999)

3. Kilbas, AA, Srivastava, HM, Trujillo, JJ: Theory and Applications of Fractional Differential Equations. North-Holland Mathematics Studies, vol. 204. Elsevier, Amsterdam (2006)

4. Samko, SG, Kilbas, AA, Marichev, Ol: Fractional Integrals and Derivations. Gordon \& Breach, Yverdon (1993)

5. Agarwal, RP, Baleanu, D, Hedayati, V, et al.: Two fractional derivative inclusion problems via integral boundary condition. Appl. Math. Comput. 257, 205-212 (2015)

6. Anderson, DR, Avery, RI: Fractional-order boundary value problem with Sturm-Liouville boundary conditions. Electron. J. Differ. Equ. 2015, 29 (2015)

7. Bai, Z, Lü, H: Positive solutions for boundary value problem of nonlinear fractional differential equation. J. Math. Anal. Appl. 311, 495-505 (2005)

8. Jia, M, Liu, X: Three nonnegative solutions for fractional differential equations with integral boundary conditions. Comput. Math. Appl. 62, 1405-1412 (2011)

9. Ntouyas, SK, Etemad, S: The existence of solutions for fractional differential inclusions with sum and integral boundary conditions. Appl. Math. Comput. 266, 235-243 (2015)

10. Yang, L, Shen, C, Xie, D: Multiple positive solutions for nonlinear boundary value problem of fractional order differential equation with the Riemann-Liouville derivative. Adv. Differ. Equ. 2014, Article ID 284 (2014)

11. Yang, L, Zhang, W, Liu, X: A sufficient condition for the existence of a positive solution for a nonlinear fractional differential equation with the Riemann-Liouville derivative. Appl. Math. Lett. 25, 1986-1992 (2012)

12. Jia, M, Liu, X: Multiplicity of solutions for integral boundary value problems of fractional differential equations with upper and lower solutions. Appl. Math. Comput. 232, 313-323 (2014)

13. Jiang, D, Yuan, C: The positive properties of the Green function for Dirichlet-type boundary value problems of nonlinear fractional differential equations and its application. Nonlinear Anal. 72, 710-719 (2010)

14. Kilbas, AA, Marzan, SA: Nonlinear differential equations with the Caputo fractional derivative in the space of continuously differentiable functions. J. Differ. Equ. 41, 84-89 (2005)

15. Li, C, Luo, X, Zhou, Y: Existence of positive solutions of the boundary value problem for nonlinear fractional differential equations. Comput. Math. Appl. 59, 1363-1375 (2010)

16. Liu, X, Jia, M, Ge, W: Multiple solutions of a p-Laplacian model involving a fractional derivative. Adv. Differ. Equ. 2013 Article ID $126(2013)$

17. Liu, X, Jia, M, Xiang, XF: On the solvability of a fractional differential equation model involving the $p$-Laplacian operator. Comput. Math. Appl. 64, 3267-3272 (2012)

18. Mohammed, A, Mohamed, AH: Monotone iterative sequences for nonlinear boundary value problems of fractional order. Nonlinear Anal. 74, 3531-3539 (2011)

19. Rida, SZ, El-Sherbiny, HM, Arafa, AAM: On the solution of the fractional nonlinear Schrödinger equation. Phys. Lett. A 372, 553-558 (2008)

20. Baleanu, D, Agarwal, RP, Mohammadi, H, et al.: Some existence results for a nonlinear fractional differential equation on partially ordered Banach spaces. Bound. Value Probl. 2013, Article ID 112 (2013)

21. Lan, KQ, Lin, W: Positive solutions of systems of Caputo fractional differential equations. Commun. Appl. Anal. 17(1), 61-86 (2013) 
22. Nyamoradi, N, Baleanu, D, Agarwal, RP: Existence and uniqueness of positive solutions to fractional boundary value problems with nonlinear boundary conditions. Adv. Differ. Equ. 2013, 266 (2013)

23. Jia, $M, L i u, X$ : The existence of positive solutions for fractional differential equations with integral and disturbance parameter in boundary conditions. Abstr. Appl. Anal. 2014, Article ID 131548 (2014)

24. Lee, MG, Lin, SS: On the positive solutions for semilinear elliptic equations on annular domain with nonhomogeneous Dirichlet boundary condition. J. Math. Anal. Appl. 181, 348-361 (1994)

25. Sun, W, Chen, S, Zhang, Q, Wang, C: Existence of positive solutions to $n$-point nonhomogeneous boundary value problems. J. Math. Anal. Appl. 330, 612-621 (2007)

26. Kong, L, Kong, Q: Second-order boundary value problems with nonhomogeneous boundary conditions I. Math. Nachr. 278(1-2), 173-193 (2005)

27. Kong, L, Kong, Q: Second-order boundary value problems with nonhomogeneous boundary conditions II. J. Math. Anal. Appl. 330, 1393-1411 (2007)

28. Kong, L, Kong, Q: Higher order boundary value problems with nonhomogeneous boundary conditions. Nonlinear Anal. 72, 240-261 (2010)

29. Guo, D, Lakshmikanthan, V: Nonlinear Problems in Abstract Cones. Notes and Reports in Mathematics in Science and Engineering, vol. 5. Academic Press, New York (1988)

\section{Submit your manuscript to a SpringerOpen ${ }^{\mathcal{O}}$ journal and benefit from:}

- Convenient online submission

- Rigorous peer review

- Immediate publication on acceptance

- Open access: articles freely available online

- High visibility within the field

- Retaining the copyright to your article 